\title{
Sexually Transmitted Infections (STIs) among young adult in Italy
}

Maria Cristina Salfa', Vincenza Regine', Laura Camoni', Mariangela Raimondo', Massimo Giuliani', Barbara Suligoi' e la Rete Nazionale di Centri clinici ${ }^{2}$ e di Laboratori ${ }^{3}$ per le Infezioni Sessualmente Trasmesse

I Centro Operativo AIDS, Dipartimento Malattie Infettive, Parassitarie e Immunomediate, Istituto Superiore di Sanità, Roma

2 Giovanni Angelini (Bari), Nicola Aste (Cagliari), Marco Cusini (Milano), Antonietta D’Antuono (Bologna), Sergio Delmonte (Torino), Aldo Di Carlo (Roma), Issa El-Hamad (Brescia), Alberto Matteelli (Brescia), Gianmichele Moise (Gorizia), Luigi Priano (Genova), Franco Urbani (Trento), Giuliano Zuccati (Firenze)

3 Giacomo Audisio (Rivoli,TO), Anna Rita Bruno (Galatina, LE), Marina Busetti (Trieste), lole Caola (Trento), Pierangelo Clerici (Legnano, MI), Carla Fontana (Roma), Cristina Giraldi (Cosenza), Maria Agnese Latino (Torino), Rosa Anna Leone (Lamezia Terme, CZ), Maria Luisa Modolo (Pordenone), Paola Pauri (Jesi,AN), Alessandra Sensini (Perugia), Luigi Tagliaferro (Lecce), con la collaborazione del Gruppo di Lavoro “Infezioni Sessualmente Trasmesse" (GLIST) dell'Associazione Microbiologi Clinici Italiani (AMCLI)

Key words: Sexually Transmitted Infections, Surveillance, Young adult

Le infezioni sessualmente trasmesse nei giovani, in Italia

\section{SUMMARY}

Sexually transmitted infections (STIs) include a large group of widespread infectious diseases, which may cause acute symptoms, chronic infections and severe long term complications. The control and prevention of these infections are public health priorities for several reasons: - the large number of people that acquire an STI per year;

- the major proportion of asymptomatic infected individuals;

- the high circulation in patients with sexual risk behavior (young adults, pluripartner, men who have sex with men, foreigners, commercial sex workers);

- increased biological susceptibility of some subjects, such as young adults (immature genital tissues and more receptive to pathogens), women (genital apparatus more complex and extended in which pathogens are more likely to settle), or individuals carrying states of severe immunodeficiency;

- the serious complications in the event of failure or incorrect diagnosis and treatment (chronic disease, infertility, oncogenic transformation, synergy with HIV infection);

- the possibility of preventing and treating many of these infections.

Therefore, recent guidelines from international agencies have recommended countries from the European Union to improve epidemiological STI surveillance systems in order to standardize data collection to facilitate their comparability between different geographical areas and to improve the information flow for faster tracking of the impact; furthermore, to extend surveillance to widespread, but often asymptomatic, disease (e.g. Chlamydia trachomatis), to conduct behavioural surveillance in patients with STIs, to increase public awareness of the role of STIs in the transmission/acquisition of HIV, and to increase the commitment of institutions in the prevention and control of STIs.

\section{INTRODUZIONE}

Le Infezioni Sessualmente Trasmesse (IST) costituiscono un vasto gruppo di malattie infettive molto diffuso in tutto il mondo, che è causa di sintomi acuti, infezioni croniche e gravi complicanze a lungo termine per milioni di persone ogni anno, e le cui cure assorbono ingenti risorse finanziarie. Secondo l'Organizzazione Mondiale della Sanità (OMS) ogni anno l'impatto delle IST corrisponde a circa 340 milioni di nuovi casi (174 milioni di nuovi casi di infezione da Trichomonas vaginalis, 92 milioni di nuovi casi di infezione da C. trachomatis, 62 milioni di nuovi casi di gonorrea, 12 milioni di nuovi casi di sifilide) tra le donne e gli uomini di età compresa tra i 15 e i 49 anni, di questi 111 milioni interessano i giovani (<25 anni). Da questo conteggio sono escluse le malattie virali, quali l'herpes genitale da Herpes simplex virus di tipo 2 (HSV-2) e le patologie genitali provocate dallo Human papillomavirus (HPV), per le quali non è facile produrre stime, ma che sono responsabili ogni anno di vari milioni di casi di infezioni nel mondo, con conseguenze sia sulla salute umana sia sullo stato socio-economico. La maggior parte delle IST si verifica nei Paesi in via di sviluppo, quali il Sud e il Sud-Est dell'Asia, l'Africa SubSahariana e l'America Latina. Anche nei Paesi più sviluppati, come l'Europa Occidentale e gli Stati Uniti, le IST sono molto diffuse (41).

Oggi si conoscono oltre 30 quadri clinici di IST determinati da circa 20 patogeni diversi, tra batteri, virus, protozoi e parassiti (41).

Il controllo e la prevenzione di queste rappresentano obietti- vi prioritari di sanità pubblica, per vari motivi (41):

- l'elevato numero di persone che ogni anno acquisisce una IST;

- la proporzione rilevante di soggetti infetti asintomatici, che può favorirne la diffusione e l'istaurarsi di complicanze;

- la più alta diffusione di queste infezioni in soggetti con comportamenti sessuali a rischio, quali soggetti con più partner, giovani, omosessuali, stranieri e persone che hanno rapporti sessuali in cambio di denaro;

- la maggiore suscettibilità biologica di alcuni soggetti, quali le donne, che presentano condizioni a livello genitale che favoriscono la colonizzazione e la virulentazione di microorganismi patogeni; gli adolescenti, che hanno tessuti genitali ancora immaturi e più recettivi ai patogeni; gli individui affetti da grave immunodeficienza;

- le gravi sequele e complicanze che si possono instaurare in caso di mancata o errata diagnosi e terapia, quali la cronicizzazione della malattia, la sterilità, la trasformazione oncogena, la sinergia con l'infezione da HIV;

- la possibilità di prevenire e curare efficacemente buona parte di queste.

Le IST fino a qualche anno fa sembravano sotto controllo, almeno nei Paesi occidentali, ma agli inizi del 2000 hanno fatto registrare in questi Paesi, Italia compresa, una recrudescenza inaspettata e mai osservata dalla fine degli anni ' 70 , soprattutto di infezioni batteriche, come sifilide, gonorrea e clamidia. Questa riemergenza ha sottolineato l'importanza di alcuni comportamenti sessuali come fattori determinanti nella diffusione di queste patologie e ha posto le condizioni

\section{Corresponding author: Maria Cristina Salfa}

Centro Operativo AIDS; Dipartimento Malattie Infettive, Parassitarie e Immunomediate Istituto Superiore di Sanità,

Viale Regina Elena, 299 - 0016 I Roma - Tel.: 0649902642 - Fax: 0649902755

E-mail: mariacristina.salfa@iss.it 
epidemiologiche per un rapido aumento anche dell'incidenza dell'infezione da $\operatorname{HIV}(5,6,9,10,11,19,30,32,34,40)$. In Italia, le informazioni disponibili sulla diffusione nazionale delle IST provengono dai dati riportati dal Ministero della Salute, che sono però limitati alle sole malattie a notifica obbligatoria, cioè gonorrea e sifilide, e che sono altresì condizionati dal problema della sottonotifica (21).

Non sono invece disponibili i dati sulla diffusione nazionale di altre IST che non sono a notifica obbligatoria, quali per esempio l'infezione da Chlamydia trachomatis $(\mathrm{Ct})$, da Trichomonas vaginalis (Tv), da Herpes simplex virus di tipo 2 (HSV-2) e da Papillomavirus umano (HPV).

Recenti direttive di organismi internazionali in tema di sorveglianza e controllo delle IST hanno raccomandato di migliorare le conoscenze sulla diffusione di queste infezioni $(3,4$, $7,16,27,28,33)$. In particolare hanno sottolineato le seguenti necessità:

- Migliorare le diagnosi delle IST e l'effettivo trattamento dei soggetti affetti utilizzando l'approccio sindromico e la diagnostica di laboratorio.

- Incrementare la consapevolezza pubblica sul ruolo delle IST nella trasmissione/acquisizione del virus dell'HIV attraverso programmi di educazione e promozione della salute sessuale (es.: utilizzo corretto e costante del condom, limitato numero di partner sessuali).

- Aumentare l'offerta dei test di diagnosi per identificare i casi asintomatici.

- Organizzare programmi di screening mirati a specifiche sottopopolazioni (es.: giovani, gravide, stranieri, omosessuali).

- Predisporre il controllo terapeutico dei soggetti e dei loro partner.

- Incrementare e facilitare l'accesso ai servizi per la diagnosi e cura.

- Potenziare la sorveglianza epidemiologica delle IST attraverso la standardizzazione della raccolta dei dati, per agevolare la loro confrontabilità tra aree geografiche diverse; il miglioramento dei flussi informativi, per un più rapido monitoraggio delle incidenze; l'affiancamento alle sorveglianze basate su centri clinici le sorveglianze basate su laboratori al fine di conoscere patologie a larga diffusione e con gravi complicanze, ma più spesso asintomatiche (es.: l'infezione da $\mathrm{Ct}$ ).

- Condurre una sorveglianza dei comportamenti nei soggetti con IST.

- Aumentare l'impegno dei governi e delle istituzione nella prevenzione e nel controllo delle IST.

- Promuovere politiche, leggi ed iniziative sul controllo delle IST (es.: screening per $\mathrm{Ct}$ ).

Per sopperire a questa mancanza di dati nazionali sulle IST e per la necessità di disporre in tempi brevi di dati sulla diffusione di queste è stato avviato, nel 1991, un Sistema di Sorveglianza Sentinella delle IST basato su centri clinici (17, $35)$.

Inoltre, per rispondere alle recenti direttive in tema di sorveglianza e controllo delle IST, e per migliorare le conoscenze sulla diffusione di queste infezioni nel nostro Paese, è stato avviato nel 2009, un Sistema di Sorveglianza basato su una rete sentinella di laboratori di microbiologia.

Viene descritta ed analizzata la metodologia specifica utilizzata per la messa a punto di questi sistemi di sorveglianza.

\section{MATERIALI E METODI}

Il sistema di sorveglianza basato su centri clinici, avviato nel 1991 sotto il coordinamento dell'Istituto Superiore di Sanità (ISS), prevede la collaborazione di una rete sentinella di 12 centri clinici pubblici, altamente specializzati nella diagnosi e nella cura dei soggetti con IST (Figura I).

Vengono segnalati i pazienti con una diagnosi clinica di IST confermata da appropriati test di laboratorio e vengono raccolte informazioni socio-demografiche e comportamentali nonché il sierostato $\operatorname{HIV}(13,14,15,36,37,38,39)$.

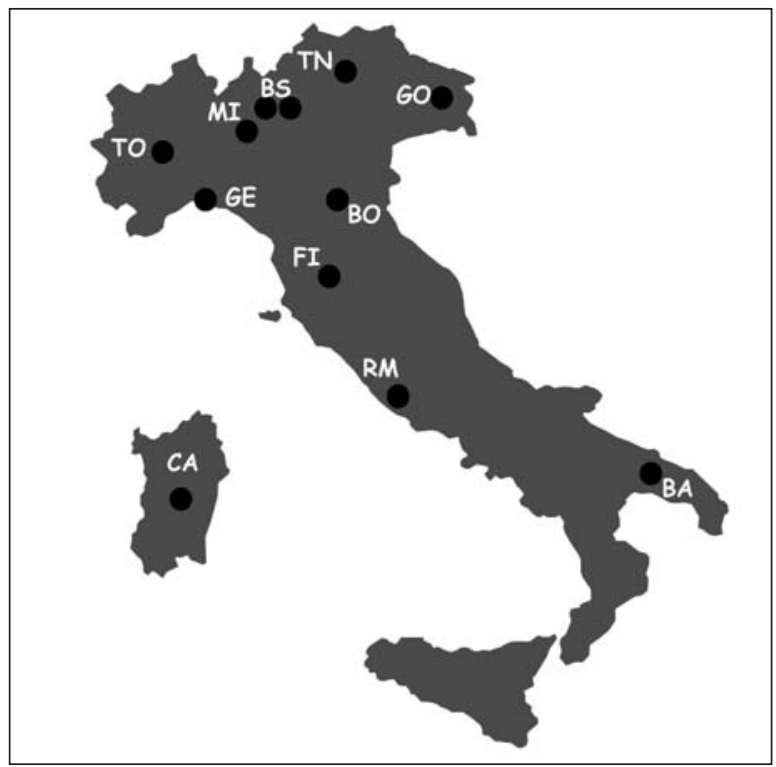

Figura I. Rete di Sorveglianza delle IST basata su 12 centri clinici pubblici.

Questo sistema raccoglie dati su numerose patologie trasmesse per via sessuale (Tabella 1); qui vengono presentati i dati relativi alle IST di maggiore rilevanza clinica.

Tabella I. IST incluse nel Sistema di Sorveglianza basato su i centri clinici, in Italia

\begin{tabular}{ll}
\hline IST & Agente eziologico \\
\hline Uretrite & N. gonorrhoeae \\
\hline Cervicite-vaginite & N. gonorrhoeae \\
\hline Proctite & N. gonorrhoeae \\
\hline Faringite & N. gonorrhoeae \\
\hline Malattia Infiammatoria Pelvica & N. gonorrhoeae \\
\hline Uretrite & C. trachomatis \\
\hline Cervicite-vaginite & C. trachomatis \\
\hline Proctite & C. trachomatis \\
\hline Faringite & C. trachomatis \\
\hline Malattia Infiammatoria Pelvica & C. trachomatis \\
\hline Uretrite & Microganismi batterici \\
& diversi da N. gonorrhoeae, \\
& C. trachomatis, T. vaginalis \\
& (Infezioni non gonococciche \\
\hline Cervicite-vaginite & non clamidiali, NG - NC) \\
\hline Proctite & Infezioni non NG - NC \\
\hline Cervicite-vaginite & Infezioni non NG - NC \\
\hline Ulcere genitali & Trichomonas vaginalis \\
\hline Ulcere genitali & C. trachomatis (LGV) \\
\hline Ulcere genitali & Calymmatobacterium \\
\hline Ulcere genitali & granulomatis \\
\hline Sifilide & Herpes simplex virus \\
\hline Sifilide & Haemophilus ducreyi \\
\hline Sifilide & T. pallidum (sifilide primaria e \\
\hline Condilomi genitali & secondaria) \\
\hline Pediculosi del pube & T. pallidum (sifilide latente) \\
\hline Mollusco contagioso & T.pallidum (reinfezione) \\
\hline & Human papillomavirus (HPV) \\
\hline & Phthirus pubis \\
\hline & Pox virus \\
\hline
\end{tabular}

Il sistema di sorveglianza basato su laboratori, avviato nel 2009 sotto il coordinamento dell'ISS e in collaborazione con il Gruppo di Lavoro "Infezioni Sessualmente Trasmesse" (GLIST) dell'Associazione Microbiologi Clinici Italiani (AMCLI), prevede la segnalazione dei nuovi casi di infezione da $\mathrm{Ct}$, da $\mathrm{Ng}$ e da Tv attraverso la collaborazione di una rete sentinella di 13 laboratori di microbiologia operanti in importanti strutture pubbliche ospedaliere o territoriali distribuite sulle tre aree principali del territorio nazionale (Nord, Centro e Sud) (Figura II) (12). 


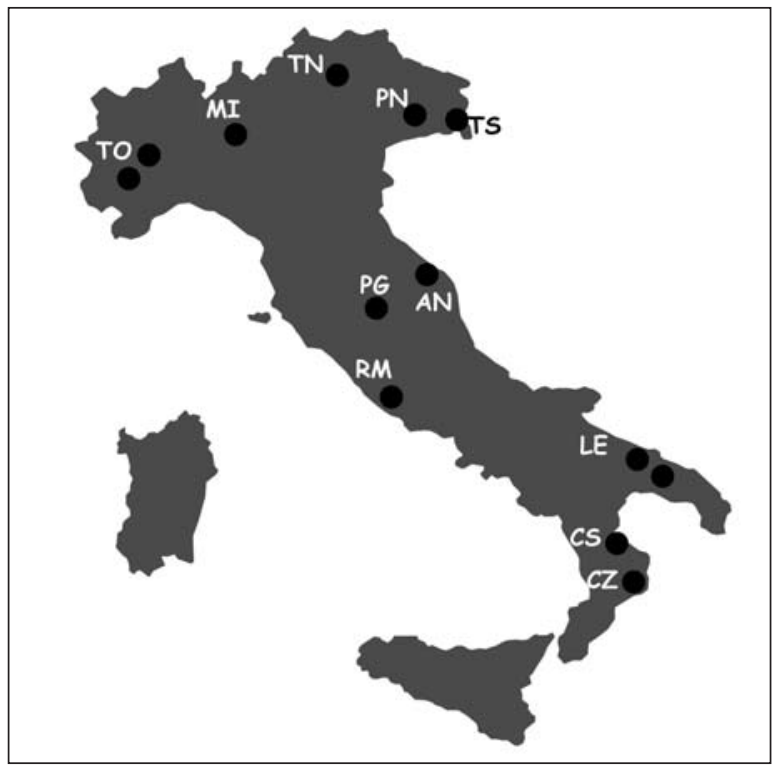

Figura II. Rete di Sorveglianza delle IST basata sui laboratori di microbiologia.

I laboratori di microbiologia raccolgono anche informazioni socio-demografiche, comportamentali e cliniche.

\section{RISULTATI}

Sistema di Sorveglianza basato sui centri clinici

Dal 1 gennaio 1991 al 31 dicembre 2007, il sistema ha raccolto un totale di 12570 nuovi casi di IST tra i giovani di età compresa tra i 14 e i 25 anni, pari al $24.7 \%$ di tutti i casi di IST segnalati al sistema nello stesso periodo.

Il $56.0 \%$ dei nuovi casi di IST tra i giovani è stato diagnosticato in uomini, il $90.4 \%$ in individui eterosessuali e il $29.6 \%$ in pazienti non italiani (la maggior parte di questi erano europei, $57.7 \%$ e africani, $24.5 \%$ )

Il $52.1 \%$ dei giovani ha riferito di avere avuto due o più partner negli ultimi sei mesi, il $12.9 \%$ di avere avuto già una IST in passato e il $2.6 \%$ ha dichiarato di aver fatto uso di droghe per via iniettiva. Relativamente all'utilizzo di metodi contraccettivi negli ultimi sei mesi, il 51.6\% degli uomini e il $46.3 \%$ delle donne ha riferito di non aver utilizzato nessun metodo contraccettivo, il $39.1 \%$ degli uomini e il $15.0 \%$ delle donne ha riferito di aver utilizzato il condom saltuariamente, il $6.3 \%$ degli uomini e 1 ' $8.6 \%$ delle donne ha riferito di aver utilizzato il condom sempre, il $27.6 \%$ delle donne ha riferito di aver utilizzato contraccettivi orali, e il 3.0\% degli uomini e il $2.5 \%$ delle donne ha riferito di aver utilizzato altri metodi contraccettivi.

L'andamento delle caratteristiche demografiche dei casi nel tempo ha mostrato una generale stabilità nella distribuzione annua dei casi per genere, mentre dalla fine degli anni ' 90 si è registrato un aumento della proporzione annua di soggetti stranieri ed omosessuali.

La proporzione di stranieri è quasi raddoppiata passando dal $23.7 \%$ (n. 142) del 1997, al 41.7\% (n. 272) del 2007, così come quella degli omosessuali che è passata dal $7.7 \%$ (n. 46) del 1997, al 12.6\% (n. 111) del 2007.

È opportuno sottolineare che anche a livello nazionale $\mathrm{i}$ permessi di soggiorno sono più che raddoppiati nello stesso periodo di tempo (circa 986000 nel 1997, circa 2400000 nel 2007).

L'andamento del numero delle segnalazioni nel tempo è rimasto costante.

Dalla distribuzione dei casi per tipo di diagnosi è emerso che le patologie più frequenti tra $\mathrm{i}$ giovani sono state $\mathrm{i}$ condilomi acuminati ano-genitali (37.2\%), le infezioni batteriche da agenti eziologici diversi da $\mathrm{Ct}, \mathrm{Ng}$ e Tv (NG-NC) (26.1\%) e le infezioni da $\mathrm{Ct}(9.7 \%)$.

La distribuzione delle patologie per genere ha mostrato tra $\mathrm{i}$
7037 giovani maschi una maggiore proporzione di condilomi acuminati ano-genitali $(42.2 \%)$ e di uretriti da $\mathrm{Ng}(12.0 \%)$, mentre tra le 5533 giovani femmine si è osservata una quota elevata di infezioni NG - NC (40.8\%) e di condilomi acuminati ano-genitali (30.9\%) (Figura III).

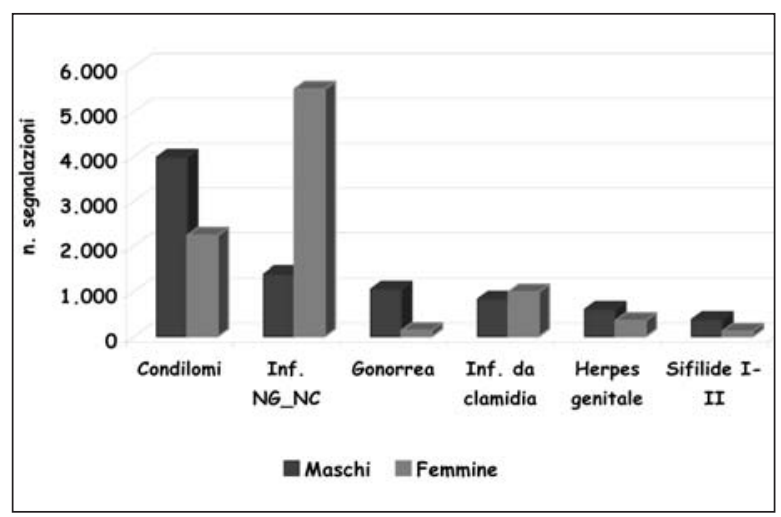

Figura III. Distribuzione delle diagnosi di IST più frequenti nei giovani ( I 4-25 anni) (Sistema di Sorveglianza Sentinella delle IST basato su centri clinici, 1991-2007).

L'andamento dei casi nel tempo e per tipo di diagnosi ha mostrato una costante riduzione di tutte le infezioni batteriche fino al 1996. Dopo il 1996 le infezioni da Ct, da Ng e la sifilide primaria e secondaria (I-II) hanno fatto registrare un aumento delle segnalazioni, con una successiva diminuzione nel 2007 (Figura IV).

$\mathrm{Al}$ contrario, il numero dei casi di infezioni NG - NC è rimasto relativamente stabile fino al 1999 , per poi diminuire progressivamente (Figura V).

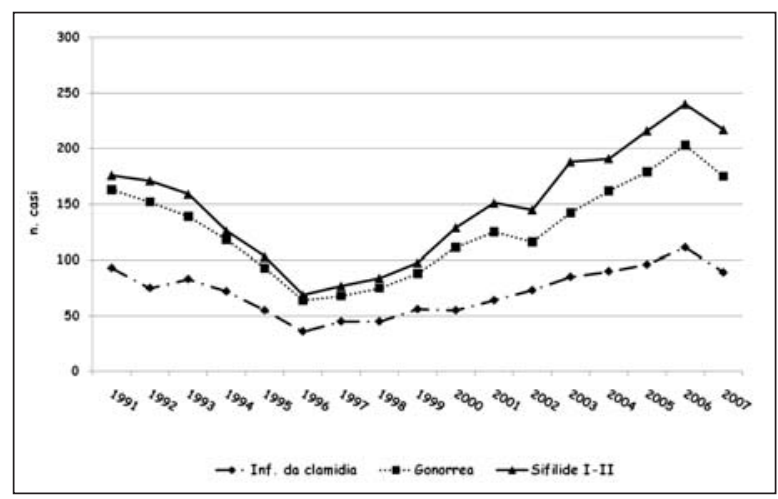

Figura IV. Andamento delle principali IST batteriche nei giovani (I4-25 anni) (Sistema di Sorveglianza Sentinella delle IST basato su centri clinici, 199/-2007).

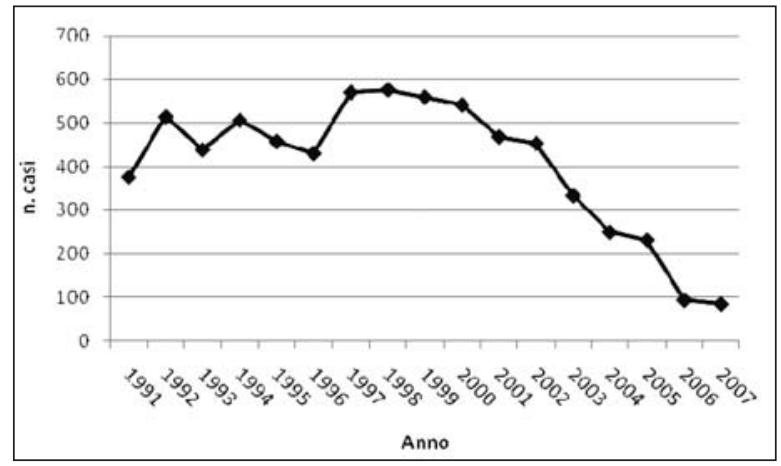

Figura V. Andamento delle infezioni NG - NC nei giovani ( $14-25$ anni) (Sistema di Sorveglianza Sentinella delle IST basato su centri clinici, 1991-2007). 
Tra le due principali IST virali, i condilomi acuminati anogenitali hanno mostrato una lieve ma costante diminuzione delle segnalazioni fino al 2005 ed un successivo incremento con un picco delle segnalazioni nel 2007, mentre l'herpes genitale ha mostrato dal 1991 al 2007 un andamento costante delle segnalazioni, che tuttavia sono state sempre numericamente inferiori rispetto a quelle dei condilomi acuminati (Figura VI).

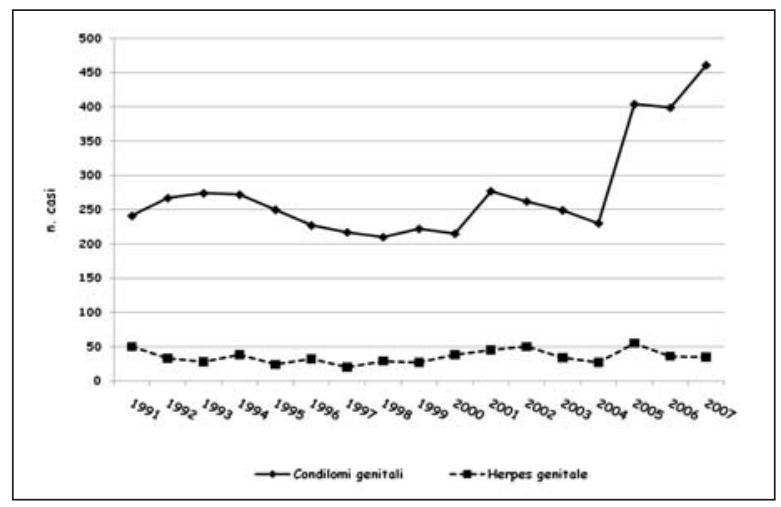

Figura VI. Andamento delle principali IST virali nei giovani ( I 4-25 anni) (Sistema di Sorveglianza Sentinella delle IST basato su centri clinici, 1991-2007).

Relativamente al sierostato per HIV, fra i 12570 casi segnalati di IST in soggetti giovani, $9552(76.0 \%)$ hanno effettuato il test anti-HIV e 271 sono risultati positivi (2.8\%). Le prevalenze di HIV hanno presentato delle differenze in base alle modalità di esposizione: la percentuale di HIV positivi era $35.8 \%$ nei soggetti che riferivano l'utilizzo di droghe per via iniettiva, $12.1 \%$ negli omosessuali, $9.0 \%$ nei bisessuali e $1.8 \%$ negli eterosessuali. La prevalenza di HIV nei maschi era lievemente più alta rispetto alle femmine ( $3.0 \%$ vs $2.6 \%)$. La prevalenza di HIV per tipo di IST era: $7.8 \%$ per sifilide I-II, $5.9 \%$ per herpes genitale, $4.7 \%$ sia per condilomi genitali che per gonorrea, $2.4 \%$ per le infezioni NG - NC e $1.3 \%$ per infezione da $\mathrm{Ct}$.

Tra i $271 \mathrm{HIV}$ positivi, il $45.4 \%$ scopriva di essere positivo in occasione del test HIV effettuato al momento della diagnosi di IST (nuove diagnosi di HIV); in particolare le nuove diagnosi di HIV erano più frequenti tra i giovani stranieri rispetto agli italiani $(75.8 \%$ vs. $35.5 \%)$.

\section{Sistema di Sorveglianza basato sui laboratori di micro-} biologia

Dal 1 aprile al 31 dicembre 2009 il sistema ha riportato dati relativi a 2021 soggetti giovani (14-25 anni) analizzati con una indicazione di approfondimento diagnostico per almeno una delle tre IST previste nella sorveglianza. I giovani rap- presentavano il $12.7 \%$ di tutti i casi segnalati a questo sistema di sorveglianza nello stesso periodo.

Il $92.4 \%$ (n. 1866) dei giovani da cui sono stati prelevati i campioni era costituito da donne e il $17.1 \%$ (n. 336) da stranieri (la maggior parte erano europei, 62.5\% e africani, $17.6 \%)$.

Il $45 \%$ dei giovani non presentava sintomi genito-urinari al momento del prelievo del campione; il 20\% delle donne era in gravidanza. Il $46.7 \%$ dei giovani riferiva di non aver utilizzato nessun metodo contraccettivo negli ultimi 6 mesi e 1' $84 \%$ dichiarava di aver avuto un solo partner sessuale negli ultimi 6 mesi.

Le prevalenze delle tre infezioni stratificate per genere e presenza o assenza di sintomi genito-urinari al momento del prelievo del campione sono mostrate nella Tabella 1. Quasi la metà delle giovani donne $(43 \%)$ con una infezione da $\mathrm{Ct}$ era asintomatica.

La prevalenza di $\mathrm{Ct}$ è risultata significativamente più elevata tra gli uomini stranieri rispetto agli italiani $(42.9 \%$ vs. $14.5 \%$, OR 4.41, 95\%CI: $1.43-13.65)$ e tra le donne, che riferivano due o più partner negli ultimi sei mesi rispetto a chi ne riferiva uno o nessuno (20.5\% vs. $5.3 \%$, OR 4.65, 95\%CI: $2.68-8.04)$.

\section{DISCUSSIONI}

Le IST interessano milioni di soggetti in tutto il mondo; in particolare $i$ giovani costituiscono una delle popolazioni più vulnerabili.

In Italia si hanno a disposizione dati sulla diffusione delle principali IST.

In particolare, $i$ dati provenienti dal sistema di sorveglianza basato su centri clinici mostrano che l'andamento del numero delle segnalazioni nei giovani (14-25 anni) è rimasto costante nel tempo.

La proporzione di giovani stranieri è quasi raddoppiata dal 1997 al 2007, ma è opportuno sottolineare che anche a livello nazionale i permessi di soggiorno sono più che raddoppiati nello stesso periodo di tempo (circa 986000 nel 1997, circa 2400000 nel 2007).

Il numero delle infezioni batteriche ha mostrato una costante riduzione fino al 1996. Dopo il 1996 le infezioni da Ct, da Ng e la sifilide I-II hanno fatto registrare un aumento delle segnalazioni, con una successiva stabilizzazione nel 2007.

L'aumento di clamidia, gonorrea e sifilide, osservato anche in altre nazioni europee (9), riflette un'incrementata circolazione di queste patologie all'inizio degli anni $2000(5,10,11)$. Questi dati mostrano anche un'elevata diffusione di condilomi genitali, cosi come riportato in altri Paesi occidentali (2, 20, 22, 24, 25, 29, 42); l'aumento rilevante di diagnosi osservato negli ultimi anni merita un attento monitoraggio nel tempo per verificare se tale tendenza si confermerà negli anni successivi.

Relativamente al sierostato HIV, più del $30 \%$ dei giovani con IST non è stato testato, nonostante l'OMS raccomandi l'effet-

Tabella I.Prevalenza delle tre infezioni per genere e per presenza o assenza di sintomi

\begin{tabular}{|c|c|c|c|}
\hline DONNE (N. I 866) & $\begin{array}{l}\text { \% positive } \\
\text { Sintomi SI* }\end{array}$ & $\begin{array}{c}\text { \% positive } \\
\text { Sintomi NO }\end{array}$ & $\begin{array}{c}\text { \% positive } \\
\text { Totali }\end{array}$ \\
\hline \multirow[t]{2}{*}{ C. trachomatis } & 7.9 & 6.5 & 7.2 \\
\hline & $57 / 726$ & $43 / 666$ & $100 / 1.392$ \\
\hline \multirow[t]{2}{*}{ T. vaginalis } & 0.3 & 0.3 & 0.3 \\
\hline & $3 / 893$ & $2 / 615$ & $5 / 1.506$ \\
\hline \multirow[t]{2}{*}{ N. gonorrhoeae } & 0.0 & 0.2 & 0.1 \\
\hline & $v 0 / 525$ & $\mathrm{I} / 545$ & $\mathrm{I} / \mathrm{I} .070$ \\
\hline \multirow[t]{2}{*}{ UOMINI (N. I 54) } & \% positivi & \% positivi & \% positivi \\
\hline & Sintomi SI* & Sintomi NO & Totali \\
\hline \multirow[t]{2}{*}{ C. trachomatis } & 22.2 & 17.0 & 20.0 \\
\hline & $16 / 72$ & $9 / 44$ & $25 / 125$ \\
\hline \multirow[t]{2}{*}{ T. vaginalis } & 0.0 & 0.0 & 0.0 \\
\hline & $0 / 40$ & $0 / 64$ & $0 / 104$ \\
\hline \multirow[t]{2}{*}{ N. gonorrhoeae } & 3.7 & 1.9 & 3.0 \\
\hline & $3 / 81$ & $\mathrm{I} / 54$ & $4 / 135$ \\
\hline
\end{tabular}

* presenza di sintomi genito-urinari al momento del prelievo del campione 
tuazione di un test $\mathrm{HIV}$ in tutti i pazienti con una diagnosi di IST (36), e quasi la metà dei giovani HIV positivi con IST ignorava il proprio sierostato costituendo, a loro insaputa, una fonte di infezione attraverso contatti sessuali non protetti o trasmissione per via parenterale. Inoltre, questi giovani a cui non era stata ancora diagnosticata l'infezione da HIV non hanno potuto usufruire di un management precoce e di cure appropriate, che possono garantire alle persone sieropositive una sopravvivenza superiore a 40 anni ed una buona qualità di vita eccellente (1).

I dati del sistema di sorveglianza basato su laboratori hanno evidenziato una maggiore diffusione di infezione da $\mathrm{Ct}$, una prevalenza di $\mathrm{Ct}$ più elevata tra i giovani uomini stranieri e tra le giovani donne pluri-partner, un'ampia quota di donne positive alla $\mathrm{Ct}$ asintomatiche.

La prevalenza di Ct osservata tra le donne giovani è particolarmente elevata, se confrontata con quella riportata in donne italiane della popolazione generale (18) e in altre nazioni dell'Europa occidentale (8, 23, 26, 31).

I dati che abbiamo raccolto indicano che la frequenza e la diffusione di queste infezioni tra i giovani in Italia è rilevante e sottolineano l'urgenza di stabilire misure di controllo da intraprendere e risorse da allocare per una adeguata prevenzione delle IST (compresa l'infezione da HIV) tra i giovani. Da quanto detto si evince che al momento attuale appare indispensabile:

- informare i giovani sulle presentazioni cliniche delle IST e sulle possibili complicanze e sequele di queste patologie;

- sensibilizzare alla necessità di rivolgersi quanto prima al proprio medico di fiducia in caso di presenza di segni o sintomi suggestivi di una IST;

- educare all'uso del condom, non solo come mezzo anticoncezionale ma anche come metodo preventivo per evitare l'acquisizione di infezioni;

- promuovere l'effettuazione del test HIV in tutti i giovani affetti da una IST;

- aumentare e facilitare l'offerta dei test di diagnosi per identificare anche i casi asintomatici.

\section{Ringraziamenti}

Gli autori ringraziano Valerio Occhiodoro per il supporto segretariale

\section{BIBLIOGRAFIA}

1. Antiretroviral Therapy Cohort Collaboration. Life expectancy of individuals on combination antiretroviral therapy in high-income countries: a collaborative analysis of 14 cohort studies. Lancet 2008; 372 (9635): 293-9.

2. Castellsangué $X$, Cohet C, Piug-Tintoré LM, et al. Epidemiology and cost of treatment of genital warts in Spain. Eur J Public Health 2008; 19: 106-10.

3. Chlamydia control in Europe. ECDC Guidance; Stockholm 2009.

4. Commission decision of 28/IV/2008 amending Decision 2002/253/EC laying down case definitions for reporting communicable diseases to the Community network under Decision No 2119/98/EC of the European Parliament and of the Council. http://ec.europa.eu/health/ph_threats/com/docs/1589_2008_en.

5. Cusini M, Ghislanzoni M, Bernardi ${ }^{-}$, et al. Syphilis outbreak in Milan, Italy. Sex Transm Infect 2004; 80 (2): 154

6. Doherty L, Fenton KA, Jones J, et al. Syphilis: old problem, new strategies. BMJ 2002; 235: 153-6.

7. Effectiveness of behavioural and psychosocial HIV/STI prevention interventions for MSM in Europe. ECDC Technical Report; Stockholm, November 2009.

8. Fenton KA, Korovessis C, Johnson AM, et al. Sexual behavior in Britain reported sexually transmitted infections and prevalent genital Chlamydia trachomatis infection. Lancet 2001; 358: 1.851-4

9. Fenton KA, Lowndes CM. Recent trends in the epidemiology of sexually transmitted infections in the European Union. Sex Transm Infect 2004; 4: 255-63.

10. Giuliani M, Di Carlo A, Dorrucci M, et al. Increased HIV incidence among men who have sex with men in Rome. AIDS 2005; 19 (13): 1429-31.

11. Giuliani M, Di Carlo A, Palamara G, Latini A, Maini A. Evidence of an outbreak of syphilis among men who have sex with men in Rome. Arch Dermatol 2005; 141 (1): 100-1.

12. Giuliani M, Salfa MC, Latino MA, Suligoi B e i Responsabili della Rete
Nazionale dei Laboratori. Una rete di laboratori pubblici per studiare le infezioni trasmesse per via sessuale. Not Ist Super Sanità 2009·2 2 (10): 3-7.

13. Giuliani M, Suligoi B, \& the STD Surveillance Working Group. Sentinel Surveillance of Sexually Transmitted Diseases in Italy. EURO Surveillance 1998; 6: 55-8

14. Giuliani M, Suligoi B, and the Italian STI Surveillance Working Group. Differences between no national and indigenous patients with sexually transmitted infections in Italy and insight into the control of sexually transmitted infections. Sex Transm Dis 2004; 31 (2): 79-84

15. Giuliani M, Suligoi B. La Sorveglianza in Italia delle Malattie Sessualmente Trasmesse. In: Malattie Sessualmente Trasmesse, Dolivo M, Orfila J, Henry-Suchet. Edizione Masson 1994; 245-55.

16. Global strategy for the prevention and control of sexually transmitted infections: 2006-2015: breaking the chain of transmission. WHO Library Cataloguing-in-Publication Data, 2007.

17. Greco D, Giuliani M, Suligoi B, et al. Sexually Transmitted Diseases in Italy. Clinical returns versus statutory notifications. Genitourin Med 1990; 66: 383-6.

18. Grio R, Bello L, Smirne C, et al. Chlamydia trachomatis prevalence in Noth-West Italy. Minerva Ginecol 2004; 56 (5): 401-6.

19. Hall HI, Song R, McKenna MT. Increases in HIV diagnoses - 29 States, 1999-2002. MMWR 2003; 47: 1145-8.

20. Hillemanns P, Breugelmans JG, Gieseking F, et al. Estimation of the incidence of genital warts and the cost of illness in Germany: a cross-sectional study. BMC Infect Dis 2008; 8: 76.

21. http://www.ministerosalute.it/malattieInfettive/paginaInternaMenuMalattie Infettive.jsp?id=812\&menu=strumentieservizi.

22. Insinga RP, Dasbach EJ, Myers ER. The health and economic burden of genital warts in a set of private health plans in the United States. Clin Infect Dis 2003; 36 (11): 1397-403

23. Klavs I, Rodrigues LC, Wellings K, Kese D, Hayes R. Prevalence of genital Chlamydia trachomatis infection in the general population of Slovenia: serious gaps in control. Sex Transm Infect 2004; 80 (2): 121-3.

24. Koutsky L. Epidemiology of genital human papillomavirus infection. Am J Med 1997; 102 (5A): 3-8

25. Kyaer SK, Tran TN, Sparen P, et al. The burden of genital warts: a study of nearly 70000 women from the general female population in the 4 Nordic countries. J Infect Dis 2007; 196: 1447-54.

26. Levidiotou S, Vrioni G, Papadogeorgaki H, Avdeliodi K, Kada H, Kaparos G. Chlamydia trachomatis infections in Greece: first prevalence study using nucleic acid amplification tests. Eur J Clin Microbiol Infect Dis 2005; 24 (3): 207-13.

27. Mapping of HIV/STI behavioural surveillance in Europe. ECDC Technical Report; Stockholm 2009.

28. Monitoring the Declaration of Commitment on HIV/AIDS. Guidelines on construction of core indicators. United Nations General Assembly Special Session on HIV/AIDS, 2010 Reporting.

29. Monsonégo J, Breugelmans JG, Bouée S, Lafuma A, Bénard S, Rémy V. Anogenital warts incidence, medical management and costs in women consulting gynaecologists in France. Gynecol Obstet Fertil 2007; 35: 107-13.

30. Nieuwenhuis RF, Ossewaarde JM, Götz HM, et al. Resurgement of lymphogranuloma venereum in western Europe: an outbreak of Chlamydia trachomatis serover L2 proctitis in the Netherlands among men who have sex with men. Clin Infect Dis 2004; 39: 996-1003.

31. Paukku M, Kilpikari R, Puolakkainen M, Oksanen H, Apter D, Paavonen J. Criteria for selective screening for Chlamydia trachomatis. Sex Transm Dis 2003; 30: 120-3.

32. Pinkock S. New HIV cases in England and Wales increase by $20 \%$ in past year. BMJ 2004; 328: 425-a.

33. Progress on Implementing the Dublin Declaration on Partnership to Fight HIV/AIDS in Europe and Central Asia. World Health Organization Regional Office for Europe, 2008.

34. Rietmeijer CA, Patnaik JL, Judson FN, Douglas JM Jr. Increases in gonorrhea and sexual risk behaviors among men who have sex with men: a 12-year trend analysis at the Denver Metro Health Clinic. Sex Trasm Dis 2003; 7: 562-7.

35. Sistema Informativo delle Malattie Infettive e Diffusive. D.M. del 15.12.1990. Gazzetta Ufficiale n. 6 del 8 gennaio 1991.

36. Suligoi B, Giuliani M and Migration Medicine Study Group: Sexually Trasmitted Diseases among foreigners in Italy. EpidemioI Infect 1997; 118 : 235-41.

37. Suligoi B, Giuliani M, Galai N, Balducci M. HIV incidence among repeat HIV testers with STD in Italy. AIDS 1999; 7: 845-50

38. Suligoi B, Giuliani M, Binkin N \& STD Surveillance Working Group. The National STD Surveillance System in Italy: Results of the first year of activity. Intern J STD \& AIDS 1994; 5: 93-100.

39. Suligoi B, Giuliani M, Binkin N and STD Surveillance Working Group. The National Surveillance System for Sexually Trasmitted Diseases in Italy. $M M W R$ 1992; 41 (SS-1): 35-41

40. van de Laar MJ, Fontaine J. ECDC guidance on Chlamydia control in Europe: next steps. Eurosurveillance 2009;14 (26): pii=19260. Disponibile all'indirizzo: ww.eurosurveillance.org/ViewArticle. aspx? ArticleId=19260.

41. WHO. Global prevalence and incidence of selected curable sexually transmitted diseases: overview and estimates. World Health Organization 2001

42. World Health Organization Regional Office for Europe. Progress on Implementing the Dublin Declaration on Partnership to Fight HIV/AIDS in Europe and Central Asia. World Health Organization 2008. 